\title{
FACTORES CRÍTICOS QUE DIFICULTAN LA APLICACIÓN DE REVENUE MANAGEMENT EN HOTELES URBANOS
}

\author{
Esther Chávez-Miranda ${ }^{1}$ \\ Departamento de Economía Financiera y Dirección de Operaciones \\ Facultad de Ciencias Económicas y Empresariales (Universidad de Sevilla) \\ e-mail: esther@us.es \\ Antonio Ruiz-Jiménez ${ }^{2}$ \\ Departamento de Economía Financiera y Dirección de Operaciones \\ Facultad de Ciencias Económicas y Empresariales (Universidad de Sevilla) \\ e-mail: anruiz@us.es \\ José Luis Pérez Díez de los Ríos ${ }^{3}$ \\ Departamento de Economía Aplicada I \\ Facultad de Ciencias Económicas y Empresariales (Universidad de Sevilla) \\ e-mail: jlperezd@us.es
}

\begin{abstract}
Resumen
Con este trabajo se pretenden determinar los factores que influyen de forma significativa como barreras para la aplicación de Revenue Management -RM- (también denominado Yield Management). Con este objetivo, se analiza la influencia de su conocimiento, implantación y de la dimensión del hotel, sobre las dificultades experimentadas por los hoteles en dos momentos, al inicio del proceso de la implantación de RM y en el momento actual. El estudio se realiza sobre el censo de hoteles de cuatro y cinco estrellas de la provincia de Sevilla. Se ha constatado, de un lado, la influencia significativa que ejerce la disponibilidad de un software de RM sobre las dificultades al inicio del proceso de implantación y, de otro, la influencia también significativa de la implantación de RM, dimensión del hotel, pertenencia a una cadena y dimensión de la cadena, sobre las dificultades a las que actualmente deben enfrentarse los hoteles para la aplicación de RM. Adicionalmente se han identificado aquellos obstáculos que resultan críticos a la hora de aplicar RM en el sector hotelero dependiendo del perfil del hotel. Con ellos el establecimiento podrá hacer una evaluación previa de sus fortalezas, debilidades y áreas de actuación prioritarias para la implantación de RM.
\end{abstract}

\section{Palabras clave}

revenue management, Yield Management, obstáculos implantación, sector hotelero

\begin{abstract}
With this work we aim to identify factors that significantly influence as barriers to the implementation of Revenue Management -RM- (also called yield management). In this way, the influence of its knowledge, its implementation and the size of the hotel is analyzed, on the difficulties experienced by hotels at two specific monents, at the beginning of the process of the implementation of $\mathrm{RM}$ and at present. The study was performed on the census of hotels of four and five star hotels in the province of Seville. It was found, on the one hand, the significant influence of the availability of a RM software on the difficulties at the beginning of the implementation process and, secondly, also significantly influence the implementation of RM, size of hotel, membership brand and size of the chain, on the difficulties they face currently hotel RM application. Additionally, those obstacles have been identified that are critical to the RM implementation in the hotel sector depending on the profile of the hotel. With them, the hotel may make a preliminary assessment of their strengths, weaknesses and areas for priority action for implementation of RM.
\end{abstract}

\section{Keywords}

revenue management, Yield Management, obstacles for implementation, hospitality sector

\section{INTRODUCCIÓN}

Revenue Management (RM) es una filosofía de gestión de éxito contrastado que persigue la obtención de la máxima rentabilidad posible del inventario perecedero (asientos de avión por milla recorrida o habitación de hotel por noche), en función de la demanda y mediante la asignación de precios. Ello se fundamenta en la utilización

\footnotetext{
${ }^{1}$ Doctora en Administración y Dirección de Empresas.

2 Doctor en Economía y Empresa.

${ }^{3}$ Doctor en Ciencias Matemáticas.
} 
de datos históricos y en la realización de previsiones (forecasting) sobre estas magnitudes, y requiere de la aplicación de técnicas que permitan que la relación entre capacidad, demanda y precios se realice de forma óptima, persiguiendo como objetivo la obtención del máximo ingreso (revenue) y/o rendimiento (yield) posibles. Sus primeras aplicaciones demostraron la percepción de beneficios económicos como el incremento de los ingresos adicionales del 2-5\% (Smith et al., 1992; Cook, 1998; Kimes, 1999; Kimes et al., 1999), de ahí que se extendiera su aplicación a sectores muy diversos en los que también se tuvo constancia de esta ventaja, como, por ejemplo, en restauración (Kimes et al., 1998; Kimes, 1999; Kimes and Thompson, 2004), empresas de alquiler de coches (Geraghty and Johnson, 1997), manufactura (Elimam \& Dodin, 2001), gestión de ventas (Siguaw et al., 2003) y gestión de proyectos (Pinder, 2005). En opinión de Cross et al. (2009) "actualmente las cadenas hoteleras líderes disponen de cientos de personas dedicadas a la definición de la estrategia, tecnología, soporte y ejecución de RM".

RM proporciona además importantes mejoras en la gestión de la empresa, como el incremento de su valor (Geraghty and Johnson, 1997), minimización de los costes de sobreocupación y ociosidad (Kasilingam, 1996), mejoras en la productividad e incrementos de la demanda (Elimam and Dodin, 2001; Kimes and Thompson, 2004), mayor utilización del inventario perecedero (Elimam and Dodin, 2001), posibilidad de obtener, de forma más rápida, el retorno de la inversión -ROI- (Lippman, 2003) y reducir la desconfianza de clientes (Siguaw et al., 2003).

La capacidad de RM para proporcionar beneficios económicos a aquellas empresas que lo han implantado, pero también no económicos (Chávez Miranda y Ruiz Jiménez, 2005a), es, sin lugar a dudas, una de las razones de su extensión. En España, son muchos los casos de implantación que pueden encontrarse, como, por ejemplo, Iberia (González-Fernández y Sulé-Alonso, 1994), grandes cadenas hoteleras (Guadix-Martín, 2004; Chávez Miranda, 2005b y Talón Ballesteros, 2009) y RENFE (González-Fernández y Sulé-Alonso, 1994). Podemos afirmar, por tanto, que actualmente continúa su proceso de consolidación en sectores tradicionales, su adaptación a sectores no tradicionales y la exploración en nuevos contextos. Por ello, aún hoy contamos con sectores no investigados, y cuya profundización permitiría hacerles partícipes de innumerables ventajas.

Centrándonos en el sector hotelero, podemos resaltar como pioneros dedicados al estudio de la implantación de RM, los trabajos desarrollados por Upchurp et al. (2002 y 2003), Luciani (1999), Jones y Hamilton (1992) y la Comisión Europea (1997). En este último grupo es en el que se incluye nuestra investigación y más concretamente en los obstáculos y/o dificultades encontrados por los hoteles para su implantación coincidiendo, por tanto, con áreas investigadas en el trabajo de la Comisión Europea (1997) y de Luciani (1999), anteriormente mencionados.

En este trabajo se analizan las dificultades que tienen los hoteles para aplicar RM. En concreto se abordarán las siguientes cuestiones:

- Dificultades que tienen los hoteles a la hora de aplicar RM en la actualidad.

- Dificultades que encontraron los hoteles que han implantado RM al comienzo del proceso de implantación.

- Diferencias entre las dificultades experimentadas por los hoteles al comienzo del proceso de implantación de RM y las que tienen en la actualidad, es decir, las dificultades superadas con la implantación de la técnica.

- Influencia de factores relacionados con la dimensión en los obstáculos iniciales y las dificultades actuales para la aplicación de RM.

- Influencia de factores relacionados con el conocimiento, la utilización de software de RM y la implantación sobre los obstáculos iniciales y las dificultades actuales para la aplicación de RM.

- Dificultades adicionales encontradas por los hoteles a la hora de aplicar RM.

- Variables críticas a tener en cuenta por el hotel a la hora de aplicar RM.

El estudio empírico se realiza sobre el censo de hoteles de cuatro y cinco estrellas de la provincia de Sevilla (España).

\section{OBJETIVOS E HIPÓTESIS DE INVESTIGACIÓN}

El propósito de esta investigación es la identificación de las variables críticas que dificultan la implantación de RM en el sector hotelero.

Para la consecución de dicho propósito u objetivo final del trabajo, se plantean los siguientes objetivos e hipótesis de investigación ${ }^{4}$ :

- 01 (Objetivo 1): Identificar los principales obstáculos que debe superar el hotel al iniciar la aplicación de RM.

- O2: Identificar las principales dificultades actuales para la aplicación de RM.

- O3: Analizar las diferencias entre los obstáculos encontrados al iniciar la aplicación de RM y las dificultades que observan en el momento actual.

\footnotetext{
${ }^{4}$ A continuación debe tenerse en cuenta que se ha utilizado el término "obstáculos" para hacer referencia a las barreras que pueden encontrar las empresas al inicio del proceso de implantación del YM para distinguirlo del término "dificultades" que se utilizará para hacer referencia a los problemas que tienen las empresas para su aplicación en el momento presente.
} 
Hipótesis $1_{[3.1]}$. Los obstáculos al inicio de la implantación difieren significativamente de las dificultades actuales.

- 04: Determinar la existencia de influencia de factores relacionados con la dimensión sobre los obstáculos al iniciar la aplicación de RM.

Hipótesis 2 a 6. La categoría del hotel $(\mathrm{H} 2[4.1])$, la dimensión del hotel $\left(\mathrm{H}_{[4.2]}\right)$, pertenencia a una cadena $\left(\mathrm{H} 4_{[4.3]}\right)$, ámbito de operación de la cadena $\left(\mathrm{H}_{[4.4]}\right)$ y dimensión de la cadena $\left(\mathrm{H} 6_{[4.5]}\right)$, individualmente considerados, influyen significativamente en los obstáculos iniciales para su aplicación.

- 05: Determinar la existencia de influencia de factores relacionados con la dimensión sobre las dificultades actuales para la aplicación de RM.

Hipótesis 7 a 11. La categoría del hotel $\left(\mathrm{H}_{[5.1]}\right)$, la dimensión del hotel $\left(\mathrm{H} 8_{[5.2]}\right)$, pertenencia a una cadena $\left(\mathrm{H}_{[5.3]}\right)$, ámbito de operación de la cadena $\left(\mathrm{H} 10_{[5.4]}\right)$ y dimensión de la cadena $\left(\mathrm{H} 11_{[5.5]}\right)$, individualmente considerados, influyen significativamente en las dificultades actuales para su aplicación.

- 06: Determinar la existencia de influencia de factores relacionados con el conocimiento e implantación de RM sobre los obstáculos al iniciar la aplicación de RM.

Hipótesis 12[6.1]. La utilización de software de RM influye significativamente en los obstáculos iniciales para su aplicación.

- 07: Determinar la existencia de influencia de factores relacionados con el conocimiento e implantación de RM sobre las dificultades actuales para la aplicación de RM.

Hipótesis 13 a 15. El conocimiento de los términos YM y RM (H13[7.1]), la implantación de RM (H14[7.2])

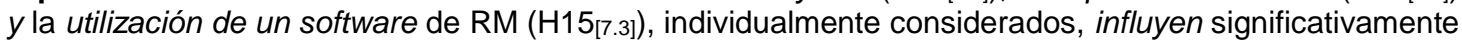
en las dificultades actuales para su aplicación.

- O8: Determinación de las variables críticas para la aplicación de RM.

\section{METODOLOGÍA}

\subsection{Población de estudio y técnica de recolección de datos}

Como propósito de la investigación nos planteamos hacer un estudio de hoteles en el caso de España. En el estudio se selecciona el censo de hoteles de Sevilla y provincia por varios motivos: (1) la limitación geográfica nos permitiría hacer un estudio en profundidad (coincidimos con Luciani, 1999), (2) el destino turístico constituye un referente a nivel nacional como ciudad de turismo cultural, junto con Madrid, Barcelona y Bilbao; (3) el nivel de respuesta obtenido podría ser superior al de otros estudios (65,2\% en Jarvis et al., 1998 y $48 \%$ en el estudio de Upchurch et al., 2002 y 2003) reduciéndose, de esta forma, el sesgo de las empresas que no contestan sobre las conclusiones obtenidas y, por último, (4) en España es frecuente la realización de estudios sobre turismo que se centren en un área geográfica determinada (Ortega Martínez y Rodríguez Herráez, 20035).

Por tanto, la población de estudio la constituye el censo de hoteles de 4 y 5 estrellas de Sevilla y provincia suponiendo un total de 45 hoteles, obteniéndose un porcentaje de respuesta del $95 \%$. El $70 \%$ de los hoteles pertenecen a cadena y, de estos, el $60 \%$ operan a nivel internacional.

Respecto a la técnica de recolección de datos encontramos que la entrevista (véase Jones y Hamilton, 1992; Jones, 1999; Comisión Europea, 1997; Luciani, 1999) y el cuestionario (por ejemplo, Jarvis et al., 1998; Comisión Europea, 1997; Luciani, 1999; Upchurch et al. 2002 y 2003) son los más utilizados en estudios similares. Además, en algunos casos la administración del cuestionario se realiza mediante entrevistas (como se puede comprobar por la coincidencia en las referencias anotadas) lo que puede resultar muy beneficioso para los resultados de la investigación no sólo porque permiten obtener mayor información (lo que enriquece sin duda una investigación descriptiva) sino también por permitir la recopilación de información cualitativa. Es por ello que en nuestro caso optamos por esta última alternativa.

Las entrevistas realizadas en la mayoría de las ocasiones se realizan al director del hotel (65\% de los casos) o a la persona indicada por éste coincidiendo con el jefe de recepción $(12,5 \%)$, el yield manager $(10 \%)$, el director comercial $(7,5 \%)$ u otros puestos (5\%). La mayoría de los entrevistados resultaron ser hombres $(57,5 \%)$.

\subsection{Consistencia interna de las escalas de medición}

Como suele ser habitual en la medición de estados subjetivos se utiliza una escala Likert de 1 a 5 puntos (coincidiendo con Upchurch et al., 2002 y 2003), donde 1 indica que el sujeto estaba totalmente de acuerdo y 5, totalmente en desacuerdo, para la medición de preguntas. Tal como aconsejan Ryan y Garland (1999) se incluye, además, la opción "no sabe/no contesta".

En lo que respecta a las escalas agregadas (Dificultades actuales para la aplicación de RM, variable "a" y Dificultades al inicio para la aplicación de RM, variable "b"), hemos de decir que todas se calculan como media aritmética de las puntuaciones de los ítems que la componen (a.1.-a.14 y b.1.-b.14., respectivamente).

\footnotetext{
${ }^{5}$ Los autores determinan, tras analizar el ámbito geográfico objeto de estudio de las tesis de turismo realizadas en España (1990-2002), que el $58.5 \%$ se dedican a una localidad, zona o área de un país.

${ }^{6}$ Sólo no contestan dos hoteles pertenecientes a la misma propiedad. No obstante, el total de encuestas cumplimentadas suman 40 porque 3 hoteles de la misma cadena, al disponer de un Revenue Manager para los tres hoteles, contestó en un único cuestionario.
} 
Para el análisis de la fiabilidad o consistencia interna de las escalas de medición se aplica el coeficiente alfa de Cronbach obteniéndose para las dificultades actuales un valor de 0.69 (14 ítems, N=20) y de 0.68 (14 ítems, $\mathrm{N}=10)$ para las dificultades al inicio. Podemos concluir, al situarse próximos al límite de referencia a utilizar $(0,7$ según Flynn et al., 1990, redondeo también aplicado por Upchurch et al., 2002 y 2003, por citar uno de los pocos trabajos en los que se hace un análisis de la consistencia interna en el área de RM), que no fue necesario eliminar ningún ítem, al alcanzarse niveles de fiabilidad interna bastante aceptables.

\subsection{Contraste de hipótesis}

El contraste de las hipótesis formuladas anteriormente nos permitirá discriminar entre las variables de estudio y, en definitiva, definir aquellas variables que se considerarán críticas para la implantación de RM.

Dados el tipo de hipótesis a contrastar, tipo de variables y número de muestras a comparar se seleccionaron los siguientes test:

- Wilcoxon para muestras relacionadas para el contraste de la hipótesis $\mathbf{H 1}$ [3.1] s. Contraste de hipótesis correlacional para medición en el caso de dos muestras ordinales.

- U de Mann Whitney para el contraste de las hipótesis H2-H15. Contraste de hipótesis de diferencia entre dos grupos.

Tanto para el tratamiento de datos como para la obtención de los estadísticos de contraste se utilizó el paquete estadístico SPSS.

\section{MARCO TEÓRICO}

Como se ha referido con anterioridad en este trabajo, son numerosas las investigaciones sobre la aplicación de $\mathrm{RM}$ en el sector hotelero e incluso a su implantación, no obstante, si nos centramos en las dificultades encontradas para su aplicación el número de trabajos disminuye notablemente.

En este ámbito resalta el estudio realizado por la Comisión Europea (1997) por realizar un acercamiento más completo y a la vez más estructurado de las dificultades para aplicar RM. El trabajo ofrece una panorámica de la aplicación de RM, en los 17 países miembros de la Comunidad Económica Europea (entre ellos España), por pequeñas y medianas empresas realizándose el análisis para distintos sectores de actividad, entre los que se incluye el sector hotelero.

La investigación dedica uno de sus apartados a los obstáculos para la aplicación de RM además de su aplicación, aplicabilidad y nivel de utilización. Ofrecen una clasificación de los obstáculos en dos categorías: factores internos (de actitud y operativos) y factores externos (de infraestructura y normativos) que, como se observará más adelante, utilizamos en nuestro estudio.

La recopilación de datos se realiza mediante entrevistas y cuestionarios a líderes en RM, líderes de empresas turísticas ${ }^{7}$ y de PYMES; y vendedores de software de RM, aunque no se hace referencia al proceso de selección de la muestra ni al número de personas que participan en el proceso.

Este trabajo también fue utilizado para el análisis de los obstáculos para la aplicación de RM por Luciani para realizar su estudio de seis hoteles de Florencia y uno de Venecia (de dos, tres y cuatro estrellas) en 1999 (p.131) exponiendo los obstáculos encontrados por los hoteles entrevistados.

Jones y Hamilton (1992) pese a dedicar este trabajo a profundizar en la aplicación de RM por los hoteles nos dejan entrever algunos obstáculos para su aplicación. Los autores identifican dos problemas en la implantación de RM: (1) la tendencia a centrarse en los detalles sin tener un conocimiento completo o claro del concepto y (2) que se presta demasiada atención a la tecnología de la información y software y, sin embargo, no se le da la merecida importancia al elemento "personas". Los autores destacan la falta de conocimiento acerca de lo que supone RM. Dicen que se conoce su aplicación a nivel táctico-operativo pero que no se tiene en cuenta el nivel estratégico. Anotan, además, la necesidad de implantar una cultura de RM. El trabajo no hace mención de las preguntas planteadas, aunque se deduce la utilización de entrevistas a directivos de hoteles y/o personal de apoyo.

También en trabajos posteriores al nuestro se analizan ítems similares a los aquí empleados. Así encontramos como Okomus (2004) también aborda, aunque de forma implícita, algunas de las dificultades anotadas. En concreto, se hace referencia a la falta de conocimiento de qué es RM, la escasa adaptación software de RM para su aplicación en el sector hotelero, el coste del software, la resistencia de directivos al proyecto, la necesidad de recursos para invertir en software y formación y la cultura de RM. El autor realiza un estudio del caso de un gran grupo hotelero (con más de 160 hoteles) de un único país. Realiza entrevistas y analiza documentos relevantes proporcionados por los hoteles.

Adicionalmente, encontramos estudios empíricos que, aunque no se centren en las dificultades para la aplicación de RM sí hacen referencia a algunos aspectos concretos, aunque de forma marginal. En concreto, la importancia de la formación ha sido argumentada en numerosos trabajos, por ejemplo, Brotherton and Mooney (1992), Donaghy et al. (1997), Kimes (2000), la formación a medida es resaltada por Donaghy et al. (1997, p. 54) o para pequeñas empresas por Normann y Mayer (1997, p. 33). La segmentación, es otro de los aspectos anotados por

${ }^{7}$ Aunque el estudio se centra en las Pymes se contacta con grandes empresas por las razones argumentadas en el trabajo (ver p. 12, Comisión Europea, 1997). 
Donaghy et al. (1995) y Upchurch et al. (2003). Respecto al software Donaghy et al. (1997, p. 54) comentan la inadecuación del software de RM actual, y Norman y Mayer (1997), por su parte, anotan el coste percibido de implantar Revenue Management System (RMS) ${ }^{8}$ como una de las causas de la lenta aplicación de RM en hoteles. La importancia de recopilación de datos históricos es resaltada por Relihan (1989, p. 45) y por Jauncy et al. (1995). Todos los aspectos anotados se incluyen de forma expresa en nuestro estudio.

Una vez realizado el análisis de los trabajos empíricos anteriores nos decantamos por el estudio de la Comisión Europea (1997) por ser el más estructurado, completo y por incluir, además, en términos generales las variables anotadas como dificultades por otros autores.

En la Tabla 1 recogemos los ítems utilizados en nuestro estudio y su correspondencia con las variables incluidas en Comisión Europea (1997) junto con la clasificación realizada en el estudio de referencia.

Tabla 1: Comparación variables utilizadas en el estudio Comisión Europea (1997) y variables de nuestro estudio

\begin{tabular}{|c|c|c|}
\hline Ítem en nuestro estudio & Variables Comisión Europea $(1997, \text { p. } 58)^{9}$ & \begin{tabular}{|c|} 
Clasificación variables \\
(Comisión Europea, \\
1997)
\end{tabular} \\
\hline No había escuchado antes nada acerca de RM & No awareness of YM $\left(^{*}\right)$ & \multirow{6}{*}{$\begin{array}{l}\text { Factores internos. } \\
\text { De actitud }\end{array}$} \\
\hline $\begin{array}{l}\text { El personal de mi empresa no ha recibido la } \\
\text { formación oportuna para aplicar la técnica }\end{array}$ & \multirow{2}{*}{$\begin{array}{l}\text { Insufficient management skills; incompatible business } \\
\text { philosophy }\end{array}$} & \\
\hline $\begin{array}{c}\text { RM no permite conseguir los objetivos establecidos a } \\
\text { nivel empresarial }\end{array}$ & & \\
\hline $\begin{array}{l}\text { Resistencia a la formalización de la información } \\
\text { (registrar toda la información de la empresa) }\end{array}$ & A resistence to formalizing information $\left({ }^{*}\right)$ & \\
\hline $\begin{array}{l}\text { RM deriva en una guerra de precios siendo por tanto } \\
\text { perjudicial para las empresas }\end{array}$ & \multirow{2}{*}{$\begin{array}{l}\text { Negative misperceptions or scepticism towards YM. } \\
\text { (1) Oversimplified understanding; (2) Association with } \\
\text { price wars \& dumping; (3) Perception that YM is only } \\
\text { for large companies }\end{array}$} & \\
\hline RM sólo se puede aplicar por las grandes empresas & & \\
\hline $\begin{array}{l}\text { RM supone un coste en términos de tecnología } \\
\text { inviable para nuestro hotel }\end{array}$ & Cost of technology (computer system) & \multirow{7}{*}{$\begin{array}{l}\text { Factores internos. } \\
\text { De operaciones }\end{array}$} \\
\hline $\begin{array}{l}\text { No es competencia del hotel es establecimiento de } \\
\text { precios }\end{array}$ & Dependence on contract business with fixed prices & \\
\hline $\begin{array}{l}\text { El hotel no dispone de información interna para } \\
\text { aplicar RM (históricos de demanda, evolución de } \\
\text { reservas realizadas a lo largo del tiempo, etc.) }\end{array}$ & \multirow[t]{2}{*}{$\begin{array}{l}\text { Insufficient business information. (Business internal } \\
\text { information; market information) }\end{array}$} & \\
\hline $\begin{array}{l}\text { El hotel no dispone de información externa (precios } \\
\text { aplicados por los competidores) para aplicar RM }\end{array}$ & & \\
\hline $\begin{array}{c}\text { El personal que se ha formado en RM, cambia de } \\
\text { puesto de trabajo y sólo transmite parte de la } \\
\text { formación recibida }\end{array}$ & High staff turnover & \\
\hline $\begin{array}{c}\text { No podemos aplicar tarifas distintas a productos o } \\
\text { servicios (habitaciones /plazas) que no se pueden } \\
\text { diferenciar }\end{array}$ & True commodity product & \\
\hline $\begin{array}{l}\text { La capacidad de nuestro hotel es demasiado } \\
\text { pequeña para aplicar RM }\end{array}$ & Capacity too small & \\
\hline \multicolumn{2}{|l|}{$\begin{array}{c}\text { En el área geográfica en la que operamos no existen } \\
\text { infraestructuras complementarias (como atracciones } \\
\text { culturales, etc) que permitan la segmentación del } \\
\text { mercado y la diversificación la demanda }\end{array}$} & \multirow{3}{*}{$\begin{array}{l}\text { Factores externos. } \\
\text { De infraestructura }\end{array}$} \\
\hline $\begin{array}{c}\text { No se dispone de un software de RM adaptado } \\
\text { totalmente al sector }\end{array}$ & $\begin{array}{c}\text { Appropiate off the shelf computer YM systems not } \\
\text { readily available }\end{array}$ & \\
\hline $\begin{array}{c}\text { Estamos sometidos a una demanda estacional } \\
\text { bastante rígida que imposibilita la segmentación del } \\
\text { mercado) }\end{array}$ & Rigid seasonality of demand & \\
\hline Restricciones legales a la variación de precios & Governmental price restrictions $\left(^{*}\right)$ & $\begin{array}{l}\text { Factores externos. } \\
\text { De regulación }\end{array}$ \\
\hline
\end{tabular}

Fuente: Elaboración propia a partir de Comisión Europea (1997, pp. 58-65)

\footnotetext{
${ }^{8}$ Con esta expresión se hace referencia al software de RM.

9 Como ya se ha referido anteriormente, los términos Yield Management (YM) y Revenue Management (RM) se utilizan indistintamente, aunque en los trabajos más recientes se utiliza de forma más extendida, por no decir, exclusiva, el segundo de
} ellos. Sin embargo, en esta columna, se utilizará YM para ser fieles al término utilizado en la fuente citada. 
Las cuestiones que aparecen con el símbolo $\left(^{*}\right)$ en la Tabla 11 fueron eliminadas durante el proceso de validación del cuestionario por los siguientes motivos:

- Resistencia a la formalización de la información porque podrían resultar comprometidas para el encuestado.

- Restricciones legales a la variación de precios. Por no ser de aplicación en nuestro país.

Este estudio presenta, por tanto, las siguientes novedades respecto a los estudios previos:

- Se revisa el listado de obstáculos contemplados en Comisión Europea (1997) adaptándolo al contexto en el que se aplica.

- Se analiza la fiabilidad o consistencia interna de las escalas de medición.

- Se realiza la medición cuantitativa de las variables en una escala Likert lo que nos permite hacer un análisis descriptivo de los resultados obtenidos y el contraste de las hipótesis de investigación.

- Se realiza un estudio transversal, es decir, en un único momento en el tiempo, de los obstáculos iniciales y las dificultades actuales para la aplicación de RM. No obstante, se asemejaría a un estudio longitudinal en tanto que nos permite comparar las dificultades experimentadas por los hoteles en el momento actual y las encontradas al principio del proceso de implantación de RM (en el caso de que el hotel hubiera implantado RM). Lo que en nuestra opinión nos permite determinar qué dificultades se superan una vez implantado RM.

- Se analiza la influencia de los siguientes factores relacionados con la dimensión en las dificultades para la aplicación de RM: categoría del hotel, dimensión del hotel (número de habitaciones), pertenencia a una cadena, ámbito de operación de la cadena (nacional e internacional), dimensión de la cadena (número de hoteles).

- Se analiza la influencia de los siguientes factores relacionados con el conocimiento y la implantación en las dificultades para la aplicación de RM: conocimiento del término YM, conocimiento del término RM, implantación de RM y utilización de un software de RM.

- Se realiza un estudio exploratorio sobre las dificultades que, aun siendo observadas por los hoteles, no se habían incorporado hasta el momento en ningún estudio.

- Se determinan las variables críticas como dificultades a tener en cuenta en función del perfil del hotel. Éste último se determinará dependiendo del valor que tengan respecto a las variables: dimensión del hotel, pertenencia a cadena, dimensión de la cadena, implantación de RM y utilización de un software de RM.

Por último, añadir, que al administrarse el cuestionario en forma de entrevista el estudio nos permite obtener información cualitativa de vital importancia para la obtención de las conclusiones de la investigación.

\section{RESULTADOS}

La presentación de resultados se realiza haciendo referencia al objetivo perseguido en cada apartado y las hipótesis contrastadas, en su caso.

\subsection{Obstáculos y dificultades para la aplicación de RM}

En este apartado expondremos los resultados obtenidos respecto a los objetivos planteados $01, \mathrm{O} 2$ y $\mathrm{O} 3$ que hacen referencia a los obstáculos encontrados antes de implantar RM (ítems contestados por aquellos hoteles que lo han implantado, de ahí que se reduzcan los $\mathrm{N}$ válidos), las dificultades encontradas por los establecimientos en el momento actual (todos los hoteles) y, por último, la comparación de los anteriores.

\section{Obstáculos iniciales}

Como se puede observar en la Tabla 2, en relación con los obstáculos iniciales y, según la opinión de los encuestados, se erigen como principales dificultades la formación del personal (valor medio de 2,1 sobre 5 ) seguido de la no disposición de información interna (media 2,9). Por tanto, resultan factores críticos a superar cuando se decide implantar RM. Para las demás variables se observan valores por encima del intermedio de la escala utilizada (3) situándose un elevado número de ítems con valores superiores a 4. 
Tabla 2: Obstáculos iniciales y dificultades actuales para la aplicación de RM. Análisis descriptivo ${ }^{10}$

\begin{tabular}{|c|c|c|c|c|c|c|c|c|}
\hline \multicolumn{5}{|c|}{ Obstáculos iniciales } & \multicolumn{4}{|c|}{ Dificultades actuales } \\
\hline Variable & & $\mathrm{N}$ válido & Media & Desv. típ. & Media & Desv. típ. & $\mathrm{N}$ válidos & Variable \\
\hline b. 1 & $\begin{array}{l}\text { El hotel no tiene competencia para el } \\
\text { establecimiento de precios }\end{array}$ & 23 & 4,6 & 0,99 & 4,9 & 0,46 & 40 & a. 1 \\
\hline b. 2 & El hotel no dispone de información interna & 23 & 2,9 & 1,79 & 4,5 & 1,06 & 40 & a.2 \\
\hline b.3 & El hotel no dispone de información externa & 23 & 3,5 & 1,62 & 4,7 & 0,64 & 40 & a.3 \\
\hline b. 4 & $\begin{array}{l}\text { No podemos aplicar tarifas distintas a habitaciones } \\
\text { que no se pueden diferenciar }\end{array}$ & 22 & 4,1 & 1,63 & 4,9 & 0,58 & 40 & a. 4 \\
\hline b. 5 & $\begin{array}{l}\text { En el área en el que operamos no hay } \\
\text { infraestructura que permitan la segmentación }\end{array}$ & 22 & 4,2 & 1,30 & 4,3 & 1,34 & 40 & a. 5 \\
\hline b. 6 & $\begin{array}{l}\text { Estamos sometidos a una demanda estacional tan } \\
\text { rígida que imposibilita la segmentación }\end{array}$ & 22 & 4,5 & 0,96 & 4,3 & 1,22 & 40 & a.6 \\
\hline b.7 & $\begin{array}{l}\text { El YM/RM no permite conseguir los objetivos } \\
\text { establecidos a nivel empresarial }\end{array}$ & 21 & 4,3 & 1,23 & 4,6 & 0,72 & 37 & a.7 \\
\hline b. 8 & $\begin{array}{l}\text { El YM/RM deriva en una guerra de precios siendo } \\
\text { perjudicial para el hotel }\end{array}$ & 21 & 3,8 & 1,61 & 4,0 & 1,34 & 37 & a. 8 \\
\hline b.9 & $\begin{array}{l}\text { El YM/RM sólo se puede aplicar a las grandes } \\
\text { empresas }\end{array}$ & 22 & 4,5 & 0,96 & 4,5 & 1,07 & 37 & a.9 \\
\hline b. 10 & $\begin{array}{l}\text { El YM/RM supone un coste en términos de } \\
\text { tecnología inviable para el hotel }\end{array}$ & 22 & 4,2 & 1,33 & 4,4 & 1,01 & 37 & a. 10 \\
\hline b.11 & $\begin{array}{l}\text { El personal del hotel no ha recibido la } \\
\text { formación oportuna para aplicar la técnica }\end{array}$ & 22 & 2,1 & 1,52 & 3,3 & 1,83 & 36 & a.11 \\
\hline b. 12 & $\begin{array}{l}\text { El personal que se forma en YM/RM cambia de } \\
\text { puesto de trabajo y sólo transmite parte de la } \\
\text { formación recibida }\end{array}$ & 22 & 4,5 & 1,06 & 4,5 & 0,97 & 36 & a.12 \\
\hline b. 13 & $\begin{array}{l}\text { La capacidad del hotel es demasiado pequeña para } \\
\text { aplicar el YM/RM }\end{array}$ & 22 & 4,8 & 0,69 & 4,4 & 1,17 & 37 & a. 13 \\
\hline b. 14 & $\begin{array}{l}\text { No se dispone de un software de YM/RM adaptado } \\
\text { totalmente al sector }\end{array}$ & 11 & 3,2 & 1,66 & 3,8 & 1,57 & 21 & a. 14 \\
\hline b. & Dificultades al inicio para la aplicación del YM/RM & 23 & 3,9 & 0,62 & 4,4 & 0,58 & 40 & a. \\
\hline
\end{tabular}

\section{Dificultades actuales}

Por otro lado, si hacemos referencia a las dificultades actuales podemos afirmar, en términos generales, que son pocas puesto que todas las variables analizadas presentan una asimetría negativa y la media de la escala agregada (variable "a" en la Tabla 2) es de 4,38.

Ahora bien, observamos que las variables en las que el entrevistado ha mostrado estar totalmente en desacuerdo (valor 5 de la escala), coinciden con las sentencias sobre su competencia para el establecimiento de precios (93\%), la aplicación de tarifas distintas a habitaciones que no se pueden diferenciar (93\%) y la disposición de información externa, es decir, de los competidores (83\%), de ahí que aparezcan con medias superiores en la Tabla 2 (4.9, 4.9 y 4.7, respectivamente). Parecen, por tanto, aspectos que no suponen ningún tipo de dificultad a la hora de aplicar la técnica.

En ocasiones, según información adicional facilitada por los entrevistados, la disponibilidad de información de los competidores, como cabía esperar, ha ido en aumento a medida que ha incrementado la utilización de la red Internet como vía para ofertar los servicios de alojamiento.

Por el contrario, encontramos que el porcentaje de hoteles que asignan una puntuación de 5 (totalmente en desacuerdo) no es tan elevado cuando se pregunta si: el personal del hotel ha recibido la formación oportuna para aplicar la técnica (47\%), existe en el mercado software de RM adaptado totalmente al sector hotelero (52\%) y que RM deriva en una guerra de precios siendo, por tanto, perjudicial para el hotel (54\%). Podríamos decir que son aspectos que aunque superados (todos ellos presentan medias superiores a 3, valor medio de la escala utilizada), suponen una mayor dificultad en tanto que presentan, comparativamente, las medias más bajas.

Si añadimos a lo anterior que las puntuaciones más bajas de la escala (valor 1), son utilizadas en la pregunta dedicada a la formación del personal, donde dicha opción es elegida por el $31 \%$ de los encuestados, y la disponibilidad de software de RM adaptado totalmente al sector, seleccionado por el 19\%, podemos concluir que parece ponerse de manifiesto la necesidad de formación sobre RM de los trabajadores de hotelería, lo que sin duda beneficiaría la implantación de RM.

En cuanto a la existencia de un software de RM para el sector hotelero los resultados son consistentes con lo indicado por Donaghy te al. (1997: 54) autores que hacían referencia a la inadecuación del software de RM en la actualidad y la necesidad de su adaptación para su aplicación en hoteles anotada por Okumus (2004: 78). De esta forma, concluimos coincidiendo con el Estudio de la Comisión Europea (1997) y con Norman y Mayer (1995) que existen oportunidades para las empresas de software. Sin embargo, la afirmación de que RM deriva en una guerra de precios nos hace pensar en un desconocimiento en profundidad sobre qué es RM o la disponibilidad

\footnotetext{
${ }^{10}$ La variable b.14 presenta un número inferior de observaciones debido a que la pregunta sólo se formulaba en el caso de que el hotel asegurase tener implantado RM pero no utilizase un software de RM.
} 
programas para el caso concreto de las PYMES. A este respecto coincidimos con aquellos autores que aseguran que en ocasiones se identifica y reduce RM a la aplicación de varias tarifas (véase Liebermann, 1993).

\section{Comparación obstáculos iniciales y dificultades actuales}

La comparación entre las dificultades encontradas por los hoteles al inicio del proceso de implantación de RM y las observadas en la actualidad nos permite obtener una imagen de su evolución en el tiempo (véase O3 e Hipótesis 1[3.1]). Estas cuestiones se plantean a los hoteles que aseguran tener implantado RM. De esta forma se pretende identificar aquellas variables que presentan diferencias significativas respecto a su medición en uno y otro momento y, en definitiva, determinar qué obstáculos se han superado con la implantación.

Como puede observarse en la Tabla 3 queda demostrada la existencia de una diferencia muy significativa entre las dificultades actuales y los obstáculos al inicio del proceso de implantación de RM con un nivel de confianza del 99\%. Además, se demuestra un comportamiento diferente en 9 de los 14 ítems analizados llegando a ser muy significativa para 3 de los obstáculos.

Tabla 3: Contraste de la Hipótesis 1[3.1.]. Las dificultades al inicio de la implantación difieren significativamente de las actuales

\begin{tabular}{|c|c|c|c|c|c|c|}
\hline \multirow{2}{*}{\multicolumn{2}{|c|}{ Variable }} & \multirow[b]{2}{*}{$\mathrm{N}$ válidos } & \multirow{2}{*}{$\begin{array}{l}\text { Media } \\
\text { Dificultades } \\
\text { actuales }\end{array}$} & \multicolumn{2}{|c|}{$\begin{array}{c}\text { Prueba de los rangos con } \\
\text { signo de Wilcoxon }\end{array}$} & \multirow{2}{*}{$\begin{array}{l}\text { Media } \\
\text { Dificultades } \\
\text { al inicio }\end{array}$} \\
\hline & & & & $\mathrm{Z}$ & Sig. Asint. & \\
\hline b1-a1 & $\begin{array}{l}\text { El hotel no tiene competencia para el } \\
\text { establecimiento de precios }\end{array}$ & 23 & $4 ., 87$ & -1.857 & 0,063 & 4,61 \\
\hline b2-a2 & El hotel no dispone de información interna & 23 & 4,61 & -3.335 & $\mathbf{0 , 0 0 1}$ & 2,87 \\
\hline b3-a3 & E hotel no dispone de información externa & 23 & 4,83 & -2.825 & 0,005 & 3,52 \\
\hline $\mathrm{b} 4-\mathrm{a} 4$ & $\begin{array}{l}\text { No podemos aplicar tarifas distintas a } \\
\text { habitaciones que no se pueden diferenciar }\end{array}$ & 23 & 5 & -2.264 & $\mathbf{0 , 0 2 4}$ & 4,09 \\
\hline b5-a5 & $\begin{array}{l}\text { En el área en el que operamos no hay } \\
\text { infraestructura que permitan la } \\
\text { segmentación }\end{array}$ & 23 & 4,74 & -2.414 & $\mathbf{0 , 0 1 6}$ & 4,18 \\
\hline b6-a6 & $\begin{array}{l}\text { Estamos sometidos a una demanda } \\
\text { estacional tan rígida que imposibilita la } \\
\text { segmentación }\end{array}$ & 11 & 4,87 & -2.041 & 0,041 & 4,45 \\
\hline b7-a7 & $\begin{array}{l}\text { El YM/RM no permite conseguir los } \\
\text { objetivos establecidos a nivel empresarial }\end{array}$ & 11 & 4,87 & -2.220 & $\mathbf{0 , 0 2 6}$ & 4,29 \\
\hline b8-a8 & $\begin{array}{l}\text { El YM/RM deriva en una guerra de precios } \\
\text { siendo perjudicial para el hotel }\end{array}$ & 11 & 4,13 & -2.041 & 0,041 & 3,76 \\
\hline b9-a9 & $\begin{array}{l}\text { El YM/RM sólo se puede aplicar a las } \\
\text { grandes empresas }\end{array}$ & 11 & 4,83 & -1.604 & 0,109 & 4,55 \\
\hline b10-a10 & $\begin{array}{l}\text { El YM/RM supone un coste en términos de } \\
\text { tecnología inviable para el hotel }\end{array}$ & 11 & 4,65 & -2.232 & 0,026 & 4,18 \\
\hline b11-a11 & $\begin{array}{l}\text { El personal del hotel no ha recibido la } \\
\text { formación oportuna para aplicar la técnica }\end{array}$ & 11 & 4,09 & -3.550 & 0,000 & 2,14 \\
\hline b12-a12 & $\begin{array}{l}\text { El personal que se forma en YM/RM } \\
\text { cambia de puesto de trabajo y sólo } \\
\text { transmite parte de la formación recibida }\end{array}$ & 11 & 4,57 & -1.000 & 0,317 & 4,5 \\
\hline b13-a13 & $\begin{array}{l}\text { La capacidad del hotel es demasiado } \\
\text { pequeña para aplicar el YM/RM }\end{array}$ & 11 & 4,87 & -1.414 & 0,157 & 4,77 \\
\hline b14-a14 & $\begin{array}{l}\text { No se dispone de un software de YM/RM } \\
\text { adaptado totalmente al sector }\end{array}$ & 11 & 4 & -1.890 & 0,059 & 3,18 \\
\hline b-a & Obstáculos o dificultades actuales & 23 & 4,66 & -4.108 & 0,000 & 3,93 \\
\hline
\end{tabular}

Sign. Asint.: Significación Asintótica (bilateral)

Así, encontramos que las dificultades actuales que presentan una diferencia muy significativa (nivel de confianza del 99\%) respecto a los obstáculos iniciales incluyen: la no disponibilidad de información interna, la no disponibilidad de información externa y la necesidad de formación. Mientras que aquéllas que presentan una diferencia significativa (margen de error del 5\%) incluyen: la no aplicación de tarifas distintas, la inexistencia de infraestructura impide la segmentación, la estacionalidad de la demanda imposibilita la segmentación, RM no 
permite la consecución de los objetivos empresariales, RM deriva en una guerra de precios, y que RM supone un coste en términos de tecnología inviable para el hotel.

Todo ello nos permite concluir que la formación y la disponibilidad de datos (tanto internos como externos) resultan cruciales como obstáculos que deben superarse al inicio del proceso por lo que deben considerarse de forma muy especial, pero además, el análisis de los datos nos permite concluir que, como era de esperar, son muchas las barreras que se superan con el proceso de implantación. La importancia de la formación y la necesidad de históricos, como ya se ha referido con anterioridad, ya se habían anotado en trabajos previos, pero a diferencia de los anteriores, en esta investigación la conclusión se obtiene tras el contraste de hipótesis de investigación.

Atendiendo a la clasificación de la Comisión Europea (1997) las variables afectadas se incluyen en todas las categorías: factores internos de actitud, factores internos de operaciones, externos de infraestructura lo que nos permite decir que se producen mejoras en todos los aspectos analizados.

\subsection{Influencia de la dimensión del establecimiento sobre los obstáculos y dificultades para aplicar RM}

A continuación expondremos los resultados obtenidos respecto a los objetivos $\mathrm{O} 4$ y $\mathrm{O} 5$ incluyendo el análisis de las hipótesis H2[4.1], H3[4.2], H4[4.3], H5[4.4], H6[4.5], respecto a los obstáculos iniciales; y de H7[5.1], H8[5.2], $\mathrm{H} 9$ [5.3], H10[5.4] yH11[5.5], en relación con las dificultades actuales.

\section{Influencia de la dimensión sobre los obstáculos iniciales}

Se recuerda que las preguntas que nos permiten la contrastación de hipótesis de este apartado fueron formuladas a aquellos hoteles que aseguraron tener implantado RM. En la Tabla 4 se pueden observar los valores obtenidos tras la aplicación del test de contraste.

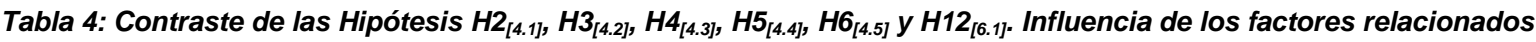
con la dimensión, de un lado, y la disponibilidad de software de RM, de otro, sobre los obstáculos al iniciar la aplicación de RM

\begin{tabular}{|c|c|c|c|c|c|c|c|c|}
\hline \multicolumn{2}{|c|}{ Test de contraste } & \multicolumn{7}{|c|}{ U de Mann-Whitney } \\
\hline \multicolumn{2}{|c|}{ Hipótes is } & & $\mathrm{H} 2_{[4.1]}$ & $\mathrm{H}_{[4.2]}$ & $\mathrm{H}_{[4.3]}$ & $\mathrm{H5} 5_{[4.4]}$ & $\mathrm{H6}_{[4.5]}$ & $\mathrm{H} 12_{[6.1]}$ \\
\hline \multirow{2}{*}{\multicolumn{2}{|c|}{ Variables }} & & 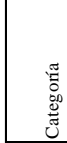 & 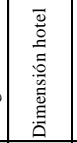 & $\underset{\tilde{\Xi}}{\tilde{J}}$ & 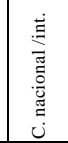 & 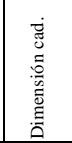 & $\begin{array}{l}\sum_{\lambda} \\
\frac{2}{\pi} \\
\sum_{0}^{2} \\
0\end{array}$ \\
\hline & & & b. 0.2 & b1.1r2 & b1.2 & $\mathrm{b} 1.3$ & b. $1.5 \mathrm{r}$ & b. 2.8 \\
\hline \multirow[b]{2}{*}{ b. 1} & \multirow{2}{*}{$\begin{array}{l}\text { El hotel no tiene competencia para el } \\
\text { establecimiento de precios }\end{array}$} & $\mathbf{U}$ & 24.000 & 42.000 & 24.000 & 37.500 & 37.500 & 63.500 \\
\hline & & Sig. E & 0,635 & 0,376 & 0,635 & 1 & 0,37 & 0,927 \\
\hline \multirow[b]{2}{*}{ b. 2} & \multirow[b]{2}{*}{ El hotel no dispone de información interna } & $\mathbf{U}$ & 20.500 & 50.500 & 14.500 & 34.000 & 40.500 & 22.000 \\
\hline & & \begin{tabular}{|l|} 
Sig. E \\
\end{tabular} & 0,404 & 0,72 & 0,166 & 0,8 & 0,503 & 0,006 \\
\hline \multirow[b]{2}{*}{ b. 3} & \multirow[b]{2}{*}{ El hotel no dispone de información externa } & $\mathbf{U}$ & 20.500 & 55.000 & 18.500 & 26.000 & 26.000 & 35.00 \\
\hline & & \begin{tabular}{|l|} 
Sig. E \\
\end{tabular} & 0,404 & 0,974 & 0,309 & 0,349 & 0,08 & 0,067 \\
\hline \multirow[b]{2}{*}{ b.4 } & \multirow{2}{*}{$\begin{array}{c}\text { No podemos aplicar tarifas distintas a } \\
\text { habitaciones que no se pueden diferenciar }\end{array}$} & $\mathbf{U}$ & 34.000 & 45.500 & 25.500 & 29.500 & 39.000 & 54.000 \\
\hline & & Sig. E. & 1 & 0,858 & 0,787 & 0,622 & 0,661 & 0,722 \\
\hline \multirow[b]{2}{*}{ b.5 } & \multirow{2}{*}{$\begin{array}{l}\text { En el área en el que operamos no hay } \\
\text { infraestructura que permitan la } \\
\text { segmentación }\end{array}$} & $\mathbf{U}$ & 15.500 & 47.000 & 27.000 & 34.500 & 38.500 & 49.500 \\
\hline & & Sig. E & 0,226 & 0,971 & 0,929 & 0,964 & 0,604 & 0,497 \\
\hline \multirow[b]{2}{*}{ b.6 } & \multirow{2}{*}{$\begin{array}{l}\text { Estamos sometidos a una demanda } \\
\text { estacional tan rígida que imposibilita la } \\
\text { segmentación } \\
\end{array}$} & $\mathbf{U}$ & 12.000 & 36.500 & 18.000 & 33.500 & 37.500 & 50.500 \\
\hline & & Sig. E & 0,132 & 0,407 & 0,356 & 0,893 & 0,549 & 0,539 \\
\hline \multirow[b]{2}{*}{ b.7 } & \multirow{2}{*}{$\begin{array}{c}\text { El YM/RM no permite conseguir los } \\
\text { objetivos establecidos a nivel empresarial }\end{array}$} & $\mathbf{U}$ & 16.500 & 25.000 & 12.500 & 28.000 & 42.000 & 51.000 \\
\hline & & Sig. E. & 0,307 & 0,24 & 0,467 & 0,559 & 0,842 & 0,809 \\
\hline \multirow[b]{2}{*}{ b. 8} & \multirow{2}{*}{$\begin{array}{l}\text { El YM/RM deriva en una guerra de } \\
\text { precios siendo perjudicial para el hotel }\end{array}$} & $\mathbf{U}$ & 24.000 & 30.500 & 6.500 & 21.000 & 35.500 & 51.000 \\
\hline & & Sig. E & 0,814 & 0,445 & 0,152 & 0,219 & 0,447 & 0,809 \\
\hline \multirow[b]{2}{*}{ b. 9} & \multirow{2}{*}{$\begin{array}{l}\text { El YM/RM sólo se puede aplicar a las } \\
\text { grandes empresas }\end{array}$} & $\mathbf{U}$ & 15.500 & 45.500 & 21.000 & 25.500 & 37.500 & 54.000 \\
\hline & & Sig.E & 0,226 & 0,858 & 0,523 & 0,391 & 0,549 & 0,722 \\
\hline \multirow[b]{2}{*}{ b. 10} & \multirow{2}{*}{$\begin{array}{l}\text { El YM/RM supone un coste en términos } \\
\text { de tecnología inviable para el hotel }\end{array}$} & $\mathbf{U}$ & 16.500 & 47.000 & 26.500 & 16.000 & 10.000 & 41.000 \\
\hline & & Sig.E & 0,265 & 0,971 & 0,857 & 0,087 & 0,003 & 0,228 \\
\hline \multirow[b]{2}{*}{ b.11 } & \multirow{2}{*}{$\begin{array}{c}\text { El personal del hotel no ha recibido la } \\
\text { formación oportuna para aplicar la técnica }\end{array}$} & $\mathbf{U}$ & 25.500 & 42.500 & 12.000 & 27.000 & 28.000 & 47.000 \\
\hline & & Sig.E & 0,787 & 0,693 & 0,132 & 0,5 & 0,182 & 0,418 \\
\hline & $\begin{array}{l}\text { El personal que se forma en YM/RM } \\
\text { cambia de puesto de trabajo y sólo }\end{array}$ & $\mathbf{U}$ & 26.500 & 45.000 & 24.000 & 25.000 & 44.000 & 42.000 \\
\hline b.12 & transmite parte de la formación recibida & Sig. E & 0,857 & 0,858 & 0,718 & 0,391 & 0,968 & 0,254 \\
\hline & La capacidad del hotel es demasiado & $\mathbf{U}$ & 24.000 & 34.500 & 22.500 & 30.000 & 35.000 & 55.500 \\
\hline b.13 & pequeña para aplicar el YM/RM & Sig. E & 0,718 & 0,329 & 0,586 & 0,687 & 0,447 & 0,771 \\
\hline & $\mathrm{YM} / \mathrm{RM}$ & $\mathbf{U}$ & 4.500 & 13.000 & 9.500 & 5.000 & 5.000 & (a) \\
\hline b.14 & adaptado totalmente al sector & Sig. E & 0,909 & 0,792 & 0,63 & 0,571 & 0,571 & \\
\hline & & $\mathbf{U}$ & 25.500 & 43.000 & 16.500 & 27.500 & 32.000 & 26.500 \\
\hline $\mathrm{b}$ & Barreras o dificultades actuales & Sig. E. & 0.698 & 0,413 & 0,23 & 0,395 & 0,201 & 0,015 \\
\hline $\mathrm{Ude}$ & e Mann-Whitney; Sign. E: Sig. Exacta; $\mathbf{c}^{2}$ : C & Chi-cuadr & drado; $\mathbf{S i}$ & ig.As.: Sig & ig. Asint & stica. & & \\
\hline (a) $[1] \mathrm{N}$ & No es posible realizar la prueba de Mann-Wh & hitney en & in grupos & s vacíos. & & & & \\
\hline & riupo $\mathrm{v}$ & & & 2 & & & & ún \\
\hline
\end{tabular}


Como se deduce de la información incluida en la tabla, se demuestra que no existe influencia de las variables agrupadas bajo la denominación de dimensión sobre los obstáculos iniciales para la aplicación de RM y, en concreto implica que la categoría del hotel, la dimensión del hotel, la pertenencia a una cadena y la dimensión de la cadena no influyen sobre los mismos.

Sin embargo, se ha podido demostrar la influencia muy significativa (asumiendo un error del 1\%) de la dimensión de la cadena en relación con la creencia de que RM supone un coste en términos de tecnología inviable para el hotel. Una vez analizados los rangos promedios de cada grupo podemos afirmar que este factor supone una mayor dificultad para aquellos hoteles que pertenecen a cadenas de hasta 50 hoteles. A nuestro juicio, ésta puede considerarse una dificultad, pero en todo caso, franqueable, pues, como ya se ha resaltado en más de una ocasión, la aplicación de RM puede ser más efectiva con la utilización de software, pero la disponibilidad del mismo no limita su adopción.

En términos generales podemos decir que destaca la diferencia que presentan los resultados de las dificultades iniciales respecto a las actuales, ya que, para estas últimas, como se verá a continuación, se ha confirmado la existencia de relación para un número superior de variables.

\section{Influencia de la dimensión sobre las dificultades actuales}

Los resultados (véase Tabla 5) indican que:

- La categoría del hotel no influye de forma significativa en las dificultades actuales para la aplicación de RM $\left(H 7_{[5.1]}\right)$. Analizando, de forma independiente, cada una de las variables que componen las dificultades actuales se ha demostrado que dicha afirmación es extensible a todas ellas, a excepción de la variable a3.8 (RM deriva en una guerra de precios resultando perjudicial para la empresa) sobre la que sí ejerce una influencia significativa. Este hecho parece razonable, en tanto que los hoteles de 5 estrellas suelen estar sometidos a una menor influencia de la bajada de precios que los de 4 estrellas; de ahí que estos últimos otorguen una mayor importancia a dicha variable. Se trata de un aspecto considerado como factor interno y, concretamente, de actitud, que supone un obstáculo para la aplicación de la técnica. Además, las condiciones del entorno económico en el que se desarrolla la investigación también podrían ser la causa de la importancia asignada a este factor, pues a lo largo del proceso de encuestación se ha hecho referencia continua, por parte de los entrevistados, a que el exceso de capacidad hotelera está provocando una bajada de precios generalizada en el sector.

- Se ha demostrado la existencia de una influencia significativa de la dimensión del hotel tanto en la escala agregada (a3), como en el ítem a3.11 (el personal no ha recibido la formación oportuna). En este caso, se confirma que los hoteles con dimensión superior a 101 habitaciones tienen menos dificultades para la aplicación de RM siendo, por tanto, aquellos con menos de 100 los más vulnerables respecto a la formación.

- La pertenencia a una cadena influye significativamente en las dificultades actuales para la aplicación de RM $\left(H 9_{[5.3]}\right)$. En cuanto al análisis detallado de los obstáculos que la conforman, se ha demostrado que la pertenencia a una cadena influye muy significativamente (obteniéndose un nivel de confianza del $99 \%$ en relación con la afirmación de que el personal no ha recibido la formación oportuna para aplicar la técnica (a3.11). Nuevamente se ha comprobado que los hoteles no pertenecientes a cadenas obtienen una menor puntuación en la variable citada, por lo que cobra especial importancia para éstos.

- El nivel en el que opera la cadena (nacional o internacional) no influye significativamente en las dificultades actuales para la aplicación de $\mathrm{RM}\left(\mathrm{H} 10_{[5.4]}\right)$. Las conclusiones obtenidas resultan, a nuestro juicio, positivas en tanto que las cadenas nacionales presentan dificultades similares a las sufridas por las que operan a nivel internacional.

- La dimensión de la cadena (medida en función del número de hoteles) influye muy significativamente (nivel de confianza del 99\%) en las dificultades actuales para la aplicación de RM (H11 [5.5]). El estudio detallado de su relación con cada una de las dificultades nos permite concluir que sólo se ha demostrado que la dimensión de la cadena influye significativamente en la creencia de que RM supone un coste, en términos de tecnología, inviable para el hotel (a3.10). Según los datos obtenidos, los hoteles pertenecientes a cadenas con menos de 51 hoteles son los que han reconocido tener más dificultades (puntuaciones tanto para la variable 3.10 , como a3, inferiores), por lo que queda demostrado que las cadenas más grandes cuentan con ventajas. 
Tabla 5: Contraste de las Hipótesis $H 7_{[5.1]}, H 8_{[5.2]}, H 9_{[5.3]}, H 1_{[5.4]}$ y $H 1_{[5.5] .}, H 1_{[7.1]}, H 14_{[7.2]}$ y H15 ${ }_{[7.3]}$. Influencia de los factores relacionados con la dimensión, el conocimiento y la implantación de RM sobre las dificultades actuales para la aplicación de RM

\begin{tabular}{|c|c|c|c|c|c|c|c|c|c|c|c|}
\hline \multicolumn{2}{|c|}{ Test de contraste } & \multirow{2}{*}{\multicolumn{10}{|c|}{ U de Mann-Whitney }} \\
\hline \multirow{2}{*}{\multicolumn{2}{|c|}{ Hipótesis }} & & & & & & & & & & \\
\hline & & & $\mathrm{H} 7[5.1]$ & $\mathrm{H} 8[5.2]$ & H9[5.3] & H10[5.4] & H11[5.5] & H13[7.1] & H13[7.1] & H14[7.2] & H15[7.3] \\
\hline \multirow{2}{*}{\multicolumn{2}{|c|}{ Variables }} & & 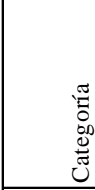 & 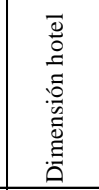 & $\begin{array}{l}\frac{\pi}{\pi} \\
\frac{\pi}{\pi} \\
\end{array}$ & 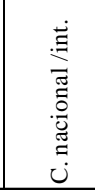 & 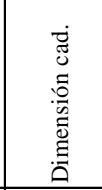 & 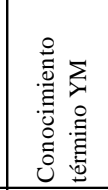 & 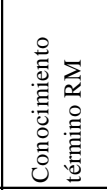 & 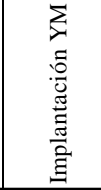 & 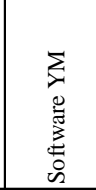 \\
\hline & & & b. 0.2 & b1.1r2 & b1.2 & b1.3 & b. $1.5 \mathrm{r}$ & b2.1 & $\mathrm{b} 2.2$ & $\mathrm{~b} 2.5$ & $\mathrm{~b} 2.8$ \\
\hline \multirow[b]{2}{*}{ a.1 } & \multirow[b]{2}{*}{$\begin{array}{l}\text { El hotel no tiene competencia para el } \\
\text { establecimiento de precios }\end{array}$} & $\mathbf{U}$ & 93.000 & 191.500 & 150.000 & 82.500 & 66.00 & 51.000 & 105.500 & 148.500 & 55.000 \\
\hline & & Sig. E & 0,754 & 0,83 & 0,61 & 0,611 & 0,374 & 0,847 & 0,728 & 0,974 & 0,563 \\
\hline \multirow[b]{2}{*}{ a. 2} & \multirow[b]{2}{*}{ El hotel no dispone de información interna } & $\mathbf{U}$ & 96.000 & 136.000 & 164.000 & 89.500 & 76.00 & 53.000 & 89.500 & 118.500 & 45.000 \\
\hline & & Sig. E & 0,839 & 0,088 & 0,919 & 0,853 & 0,705 & 0,923 & 0,363 & 0,312 & 0,232 \\
\hline \multirow[b]{2}{*}{ a. 3} & \multirow[b]{2}{*}{ El hotel no dispone de información externa } & $\mathbf{U}$ & 99.000 & 147.500 & 162.000 & 92.500 & 82.00 & 48.000 & 61.000 & 129.000 & 55.000 \\
\hline & & Sig. E & 0,926 & 0,161 & 0,873 & 0,963 & 0,94 & 0,734 & 0,053 & 0,515 & 0,563 \\
\hline \multirow[b]{2}{*}{ a.4 } & \multirow{2}{*}{$\begin{array}{l}\text { No podemos aplicar tarifas distintas a } \\
\text { habitaciones que no se pueden diferencia }\end{array}$} & $\mathbf{U}$ & 93.000 & 168.000 & 146.000 & 85.000 & 78.00 & 39.000 & 66.000 & 138.000 & 65.000 \\
\hline & & Sig. E & 0,754 & 0,405 & 0,531 & 0,711 & 0,781 & 0,43 & 0,081 & 0,721 & 1 \\
\hline \multirow[b]{2}{*}{ a. 5} & \multirow{2}{*}{$\begin{array}{c}\text { En el área en el que operamos no hay } \\
\text { infraestructura que permitan la } \\
\text { segmentación }\end{array}$} & $\mathbf{U}$ & 96.000 & 154.000 & 147.000 & 80.500 & 72.00 & 50.500 & 83.500 & 107.500 & 60.500 \\
\hline & & Sig. E. & 0,839 & 0,226 & 0,55 & 0,547 & 0,56 & 0,809 & 0,261 & 0,169 & 0,784 \\
\hline \multirow[b]{2}{*}{ a. 6} & \multirow{2}{*}{$\begin{array}{l}\text { Estamos sometidos a una demanda } \\
\text { estacional tan rígida que imposibilita la } \\
\text { segmentación }\end{array}$} & $\mathbf{U}$ & 79.500 & 135.000 & 155.500 & 67.000 & 66.00 & 55.000 & 93.000 & 69.500 & 57.000 \\
\hline & & Sig. E & 0,404 & 0,083 & 0,716 & 0,225 & 0,374 & 1 & 0,442 & $\mathbf{0 , 0 0 7}$ & 0,648 \\
\hline \multirow[b]{2}{*}{ a.7 } & \multirow{2}{*}{$\begin{array}{c}\text { El YM/RM no permite conseguir los } \\
\text { objetivos establecidos a nivel empresarial }\end{array}$} & $\mathbf{U}$ & 63.000 & 165.500 & 108.000 & 73.000 & 60.00 & (a) & 52.000 & 95.500 & 61.500 \\
\hline & & Sig. E & 0,231 & 0,892 & 0,371 & 0,57 & 0,347 & & 0,524 & 0,075 & 0,832 \\
\hline \multirow[b]{2}{*}{ a. 8} & \multirow{2}{*}{$\begin{array}{l}\text { El YM/RM deriva en una guerra de } \\
\text { precios siendo perjudicial para el hotel }\end{array}$} & $\mathbf{U}$ & 42.000 & 148.500 & 80.000 & 49.000 & 49.50 & (a) & 65.000 & 119.000 & 60.500 \\
\hline & & Sig. E & $\mathbf{0 , 0 3 5}$ & 0,517 & 0,062 & 0,074 & 0,123 & & 0,981 & 0,328 & 0,784 \\
\hline \multirow[b]{2}{*}{ a. } & \multirow{2}{*}{$\begin{array}{c}\text { El YM/RM sólo se puede aplicar a las } \\
\text { grandes empresas }\end{array}$} & $\mathbf{U}$ & 81.000 & 151.000 & 100.500 & 85.000 & 61.50 & (a) & 64.000 & 106.000 & 61.000 \\
\hline & & Sig. E & 0,644 & 0,577 & 0,242 & 1 & 0,376 & & 0,944 & 0,159 & 0,832 \\
\hline \multirow[b]{2}{*}{ a. 10} & \multirow{2}{*}{$\begin{array}{c}\text { El YM/RM supone un coste en términos } \\
\text { de tecnología inviable para el hotel }\end{array}$} & $\mathbf{U}$ & 54.000 & 141.000 & 101.500 & 71.000 & 36.00 & (a) & 48.000 & 85.500 & 55.500 \\
\hline & & Sig. E & 0,114 & 0,39 & 0,257 & 0,505 & $\mathbf{0 , 0 2 2}$ & & 0,407 & $\mathbf{0 , 0 3 4}$ & 0,563 \\
\hline \multirow{2}{*}{ a.11 } & \multirow{4}{*}{$\begin{array}{c}\text { El personal del hotel no ha recibido la } \\
\text { formación oportuna para aplicar la técnica } \\
\text { El personal que se forma en YM/RM } \\
\text { cambia de puesto de trabajo y sólo } \\
\text { transmite parte de la formación recibida }\end{array}$} & $\mathbf{U}$ & 81.000 & 86.000 & 43.000 & 57.000 & 47.50 & (a) & 44.000 & 44.000 & 38.500 \\
\hline & & Sig. E & 0,725 & 0,018 & $\mathbf{0 , 0 1}$ & 0,241 & 0,16 & & 0,34 & $\mathbf{0 , 0 0 1}$ & 0,101 \\
\hline & & $\mathbf{U}$ & 86.500 & 133.000 & 115.000 & 82.000 & 56.50 & (a) & 46.000 & 114.000 & 51.000 \\
\hline a. 12 & & Sig. E. & 0,885 & 0,404 & 0,83 & 0,902 & 0,247 & & 0,872 & 0,253 & 0,41 \\
\hline & La capacidad del hotel es demasiado & $\mathbf{U}$ & 81.000 & 117.000 & 105.500 & 71.500 & 54.00 & (a) & 64.000 & 89.500 & 55.000 \\
\hline a.13 & pequeña para aplicar el YM/RM & Sig. E. & 0,644 & 0,11 & 0,319 & 0,505 & 0,205 & & 0,944 & 0,047 & 0,563 \\
\hline & No se dispone de un software de YM/RM & $\mathbf{U}$ & 30.500 & 43.500 & 45.000 & 13.500 & 9.50 & (a) & 15.500 & 43.000 & 36.500 \\
\hline a.14 & adaptado totalmente al sector & Sig. E & 0,763 & 0,464 & 0,554 & 0,485 & 0,412 & & 0,686 & 0,656 & 0,077 \\
\hline & & $\mathbf{U}$ & 94.500 & 106.000 & 99.500 & 59.500 & 33.00 & 38.000 & 101.500 & 34.000 & 36.500 \\
\hline a & Dificultades actuales & Sig. E. & 0,782 & 0,011 & 0,042 & 0,111 & 0,008 & 0,401 & 0,626 & $\mathbf{0 , 0 0 0}$ & 0,077 \\
\hline
\end{tabular}

U: U de Mann-Whitney; Sign. E: Sig. Exacta; $\mathbf{c}^{2}$ : Chi-cuadrado; Sig.As.: Sig. Asintótica.

(a)[1] No es posible realizar la prueba de Mann-Whitney en grupos vacíos.

[1] La pregunta "a.14" se administraba a aquellos que aseguraban no utilizar ningún software de YM, es decir, respondían "no" al ítem "b2.8", de ahí que en este punto aparezca un grupo vacío.

\subsection{Influencia del conocimiento e implantación de RM sobre los obstáculos y dificultades para su aplicación}

Son objeto de análisis en este apartado los objetivos $\mathrm{O} 6, \mathrm{O} 7$ y el contraste de las hipótesis $\mathrm{H} 12$ [6.1], $\mathrm{H} 13_{[7.1]}$,

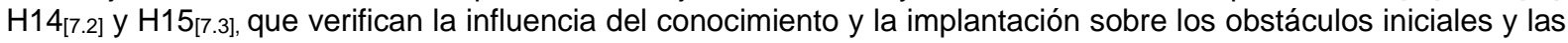
dificultades actuales.

\section{Influencia del conocimiento y la disponibilidad de software de RM sobre los obstáculos iniciales}

Tal como se puede interpretar, una vez observados los valores recogidos en la Tabla 4, la utilización de software de RM influye significativamente en los obstáculos iniciales para su aplicación. De esta forma, queda demostrado que, aunque la aplicación de RM no exija la disponibilidad de un programa informático, este hecho sí facilita el proceso de implantación, en tanto que disminuyen, de forma importante, las dificultades encontradas al inicio de dicho proceso. 
Por otro lado, revisando cada una de las dificultades objeto de estudio, sólo se ha demostrado la influencia, con un nivel de confianza del $99 \%$, de la utilización de software de RM sobre la disponibilidad de información interna (b3.2). Los hoteles que dicen utilizar algún programa muestran un mayor grado de acuerdo con el ítem b3.2 que aquellos otros que no lo utilizan. A este respecto, la recogida de datos evidenció que cuando el entrevistado hacía referencia a software de RM no tenía por qué tratarse de un programa informático específicamente diseñado y dedicado a la actividad de RM, sino que también se refiere a la utilización de hojas de cálculo de MsExcel como apoyo para la adopción de decisiones en materia de RM.

Esta relación es de gran relevancia, ya que demuestra la importancia de la disposición de datos internos, como paso previo a la implantación de la técnica, en el caso de utilizar software de RM.

\section{Influencia del conocimiento y la implantación de RM en las dificultades actuales}

Según marca la lógica, las dificultades que observan los hoteles que actualmente han implantado RM deben diferir de forma significativa respecto a las que tienen los que no lo han hecho. No obstante, lo que se pretende con el contraste de la hipótesis planteada es, de un lado, confirmar científicamente esta afirmación y, de otro, identificar qué variables presentan diferencias significativas cuando comparamos el grupo de hoteles que afirman tener implantado RM con los que niegan haberlo hecho.

La información incluida en la Tabla 5 nos permite confirmar una diferencia muy significativa entre los valores manifestados por los hoteles que han implantado RM y los que no lo han hecho. Pero, analicemos también qué ha ocurrido en lo que al comportamiento individual de cada ítem.

Destaca, una vez más, la diferencia muy significativa de la formación (con un margen de error del $1 \%$ ). Sin embargo, en este caso, surgen nuevos obstáculos que deben ser considerados: con influencia también muy significativa, la estacionalidad de la demanda como factor influyente en la segmentación (nivel de confianza del 99\%) y de forma significativa (nivel de confianza del 95\%) el coste de la tecnología y la capacidad del hotel.

Se ha comprobado, además, tras analizar el rango promedio de las puntuaciones obtenidas en el caso de tener 0 no tener implantada la técnica, que en todos los casos descritos, los hoteles que no han implantado RM aparecen como más vulnerables a estas dificultades. Los resultados obtenidos apuntan a que las anotadas anteriormente, son dificultades que se han superado por aquellos hoteles que aseguran haber implantado RM.

La mayor dificultad para segmentar el mercado derivada de la estacionalidad de la demanda ha sido demostrada, aunque no en el contexto que ahora analizamos, por Jarvis et al. (1998). Sin embargo, en nuestro caso, podemos decir que al no demostrarse la influencia significativa sobre esta variable de otros aspectos, como los relativos a la dimensión, nos encontramos con que empresas con un número de habitaciones similares (que podrían tener los mismos problemas a la hora de afrontar la segmentación), han superado esta dificultad cuando se ha implantado RM. Es por ello que, en nuestra opinión, se trata más de una barrera de actitud relacionada con la falta de formación suficiente sobre RM.

En el mismo sentido explicamos la relación con la variable referente a que la capacidad del hotel es demasiado pequeña para aplicar la técnica, puesto que no se ha podido demostrar la influencia de la dimensión sobre este aspecto.

Respecto a la influencia confirmada de la implantación sobre la impresión de que RM supone un coste en tecnología que resulta inviable para el hotel, hemos de recordar que dicha implantación no exige la disponibilidad de software, hecho que, a la vista de los resultados, parece ser reconocido por aquellos hoteles que lo han implantado, de ahí las diferencias observadas.

\subsection{Dificultades adicionales}

En este apartado nos detenemos a analizar los resultados obtenidos respecto a la formulación de una pregunta abierta a los encuestados sobre lo que en su opinión podrían considerarse como obstáculos al inicio y/o dificultades actuales adicionales a los incluidos en el cuestionario administrado.

Entendemos que la importancia de este apartado radica en la posibilidad de ampliar los ítems del estudio de la Comisión Europea, incluyendo aquellos que han identificado los propios encuestados lo que beneficiará a futuras investigaciones al respecto.

Lo aquí anotado refleja la opinión personal y la impresión de los entrevistados de ahí que a veces se repitan algunos ítems que ya se habían incluido en el cuestionario (aparecen en negrita en la Tabla 6). Hemos de destacar el hecho de que, como se puede comprobar, en general, dichos aspectos coinciden con los que se han erigido como más importantes en el análisis realizado anteriormente.

Tabla 6: Barreras y dificultades, actuales y al inicio, para la aplicación de RM adicionales a las incluidas en el estudio de Comisión Europea (1997)

\begin{tabular}{|c|c|c|}
\hline \multicolumn{2}{|l|}{ Factores internos } & Factores externos \\
\hline De actitud (D.A.) & De operaciones (D.O.) & De infraestructura (D.I.) \\
\hline $\begin{array}{l}\text { Resistencia al cambio. } \\
\text { Cambio de cultura. } \\
\text { Necesidad de formación. } \\
\text { Desconfianza por parte del cliente. } \\
\text { Desconocimiento. } \\
\text { Cuando la oferta supera la demanda: } \\
\text { tendencia a la disminución de precios. } \\
\text { Falta de información en español. }\end{array}$ & $\begin{array}{l}\text { Necesidad de planificar. } \\
\text { Rotación de personal. } \\
\text { No disponibilidad de históricos de } \\
\text { precios. } \\
\text { Exige actualización continua de datos, } \\
\text { contemplando nuevas situaciones no } \\
\text { previstas. } \\
\text { Históricos no fiables. }\end{array}$ & $\begin{array}{l}\text { Complejidad del software. } \\
\text { Inexistencia de software. } \\
\text { Inexistencia de software para PYMES } \\
\text { exige aplicación manual. } \\
\text { Localización del hotel. } \\
\text { Software. } \\
\text { Problemas informáticos. }\end{array}$ \\
\hline
\end{tabular}




\begin{tabular}{|l|l|l|}
\hline & Exige revisiones continuas al principio. & \\
& Se necesita más tiempo para controlar. & \\
& No se dispone de personal con dedicación & \\
& exclusiva. & \\
Falta de tiempo. & \\
\hline
\end{tabular}

Para su análisis, se procedió a la agrupación de las respuestas ofrecidas conforme a la clasificación incluida en el estudio de la Comisión Europea (1997) que distingue entre: factores internos de actitud (D.A.) y de operaciones (D.O.), de un lado, y factores externos de infraestructura (D.I.), de otro.

Con respecto a las aportaciones realizadas por los entrevistados nos gustaría destacar lo siguiente:

Factores internos. De actitud. Encontramos que algunas de las dimensiones citadas ya se incluían en los estudios de algunos autores a nivel académico. Así, en algunos trabajos se hace referencia a la necesidad de formación (Jones y Hamilton, 1992; Jarvis et al., 1998; Jones, 1999); Donaghy et al. (1997) mencionan la gestión del cambio y la desconfianza de los clientes, derivada de la aplicación de tarifas múltiples para el mismo servicio, es abordada en numerosas ocasiones por Kimes (1994 y 2002); e incluso el estudio de Comisión Europea (1997) hacía referencia a aspectos relativos a la necesidad de difusión de la información relativa al RM. Por el contrario, resulta novedosa la alusión al exceso de capacidad sobre los precios. Este factor preocupa sobremanera a los profesionales del sector en la actualidad y podría ser consecuencia de un conocimiento limitado sobre RM, ya que con frecuencia se piensa que la aplicación de RM se reduce a la aplicación de descuentos sobre el precio.

Factores internos. De operaciones. Los distintos aspectos anotados pueden agruparse en dos apartados, necesidad de planificación, de un lado, y control continuo de los registros (ya sean históricos y actuales), de otro, lo que exige disponibilidad de tiempo y personal adecuado que además sea responsable de estas actividades. No obstante, la información recopilada, nos ha permitido observar que no existe unanimidad respecto a la necesidad de personal adicional, cuando sí existe, como era de esperar, en torno a la disponibilidad de históricos fiables. Estos últimos se erigen como uno de los pilares básicos para la aplicación de RM. La fiabilidad de los datos viene determinada no sólo por la calidad y cantidad de los registros, sino también, por la disponibilidad de todos los datos necesarios (variedad). No en vano, en nuestro trabajo ya se ha hecho referencia a la necesidad de recoger, tanto datos internos como externos.

Factores externos. De infraestructura. La mayoría de las razones incluidas en este punto hacen referencia directa al software, a excepción de la localización del hotel. Al primero de los aspectos ya se ha dedicado un apartado, por lo que no estimamos necesario hacer ninguna anotación adicional, mientras que al segundo se hace alusión en trabajos previos como el de Jarvis et al. (1998).

\section{VARIABLES CRÍTICAS PARA LA APLICACIÓN DE RM EN FUNCIÓN DEL PERFIL DEL HOTEL}

Derivado del análisis realizado y del contraste de las hipótesis de investigación formuladas estamos en condiciones de determinar qué variables pueden considerarse críticas a la hora de aplicar RM. Para ello hemos confeccionado una tabla donde se recogen, de forma resumida, los resultados obtenidos con la investigación realizada (ver Tabla 7).

Tabla 7: Variables críticas para la aplicación de RM en función del perfil del hotel.

\begin{tabular}{|c|c|c|c|}
\hline Perfil del hotel & Variables críticas & $\begin{array}{l}\text { Variables adicionales si el } \\
\text { hotel NO dispone de } \\
\text { software de RM }\end{array}$ & $\begin{array}{l}\text { Variables adicionales si el } \\
\text { hotel no tiene implantado } \\
\text { RM }\end{array}$ \\
\hline $\begin{array}{l}\text { Hotel independiente } \\
<100 \text { habitaciones }\end{array}$ & $\begin{array}{l}\text { Este hotel es el que deberá } \\
\text { enfrentarse a mayores } \\
\text { dificultades para la aplicación de } \\
\text { RM. La formación se convierte } \\
\text { en esencial e imprescindible } \\
\text { erigiéndose como variable crítica. } \\
\text { Se recomienda... }\end{array}$ & \multirow{4}{*}{$\begin{array}{l}\text { Estos hoteles presentan más } \\
\text { dificultades al inicio, en } \\
\text { general, y respecto a la } \\
\text { disponibilidad de información } \\
\text { interna, en particular. }\end{array}$} & \multirow{4}{*}{$\begin{array}{l}\text { Deberá prestarse una atención } \\
\text { especial a la influencia de la } \\
\text { demanda estacional en la } \\
\text { segmentación, el coste de la } \\
\text { tecnología, la formación y la } \\
\text { capacidad del hotel. }\end{array}$} \\
\hline $\begin{array}{l}\text { Hotel independiente } \\
\geq 100 \text { habitaciones }\end{array}$ & $\begin{array}{l}\text { Se sitúa en segundo lugar } \\
\text { atendiendo a las dificultades para } \\
\text { la aplicación de RM. También } \\
\text { deberá prestar una atención } \\
\text { especial a la formación, aunque } \\
\text { su dimensión hace que esta } \\
\text { necesidad no sea tan acuciante } \\
\text { como en el caso anterior. }\end{array}$ & & \\
\hline $\begin{array}{l}\text { Hotel } \\
<100 \text { habitaciones } \\
\text { perteneciente a cadena } \\
<50 \text { hoteles }\end{array}$ & $\begin{array}{l}\text { Se sitúa en tercer lugar. En este } \\
\text { caso el coste de la tecnología } \\
\text { será el factor crítico al que se le } \\
\text { debe dedicar más atención junto } \\
\text { con la formación. }\end{array}$ & & \\
\hline $\begin{array}{l}\text { Hotel } \\
\geq 100 \text { habitaciones } \\
\text { perteneciente a cadena }\end{array}$ & $\begin{array}{l}\text { Para los hoteles que formen parte } \\
\text { de este grupo el factor más } \\
\text { importante es el coste de la }\end{array}$ & & \\
\hline
\end{tabular}




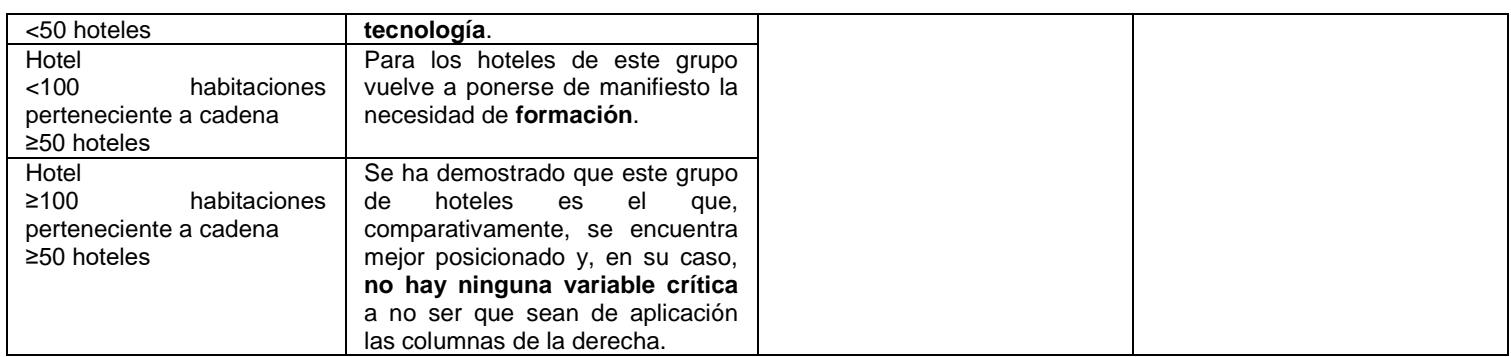

La importancia de la tabla aportada radica en el hecho de que cada hotel, utilizando la misma, podrá evaluar qué factores le pueden influir dependiendo de su perfil, de forma especial. El perfil del hotel se determinará en base a las variables analizadas en nuestro estudio respondiendo, en concreto, a las siguientes cuestiones: dimensión del hotel, pertenencia a una cadena y la dimensión de la cadena. Las variables críticas se incluyen en la columna correspondiente. No obstante, si el hotel no dispone de software de RM o no tiene implantado RM deberá considerar, además de las variables anotadas en su caso en concreto (columna 2), las incluidas en las columnas tercera y cuarta de la tabla. Los distintos perfiles de hotel aparecen ordenados en la primera columna desde los que presentan mayores dificultades (primeras posiciones) hasta el que no ha observado ninguna dificultad (última fila en la tabla).

Dado que ni la categoría del hotel ni el ámbito de operación de la cadena han demostrado ejercer influencia en las variables analizadas no se han considerado para la determinación del perfil del hotel y, por tanto, no se han incorporado en la Tabla 7.

A modo de ejemplo podríamos decir que un hotel pequeño (de hasta 100 habitaciones) e independiente que se propone la implantación de RM, forma parte del grupo que presenta más dificultades. Deberá prestar una atención especial a la formación resultando aconsejable, además, que adquiera un software de RM siempre que supere el obstáculo de disponibilidad de información interna. Mientras que para un hotel perteneciente a una cadena pequeña (de hasta 50 hoteles) el coste de la inversión se convierte en un factor importante a tener en cuenta tanto al inicio del proceso de implantación como una vez implantado.

Los resultados demuestran que los hoteles grandes (con más de 100 habitaciones) pertenecientes a cadenas constituidas por más de 50 hoteles que tienen implantado RM y que, además, disponen de un software de RM son los menos vulnerables.

\section{CONCLUSIONES}

Como aspectos destacables de las hipótesis contrastadas podemos decir que, a nuestro parecer, los resultados obtenidos son especialmente importantes y dignos de tener en cuenta por parte de los hoteles independientes, de pequeña o mediana dimensión (con menos de 100 habitaciones), así como por aquellos otros que pertenecen a cadenas cuyo número de establecimientos no supera los cincuenta, puesto que en estas situaciones se ha demostrado que aumentan las dificultades que se pueden encontrar a la hora de aplicar RM.

Además, con el estudio realizado hemos podido comprobar cuáles son los factores a los que dichas empresas deberán prestar una atención especial. Como vías para reducir la influencia de dichos factores proponemos la agrupación de los hoteles, de un lado, y la promoción de cursos de formación dirigidos al personal de estas empresas por iniciativa pública, de otro. Aconsejamos estas dos medidas basándonos en la limitación de recursos de los hoteles que han resultado más vulnerables, esto es, los de menor dimensión.

En la Figura 1 se incluyen las variables para las que se ha demostrado que ejercen una influencia significativa ( $5 \%$ de error) o muy significativa (1\%) sobre los obstáculos iniciales y las dificultades actuales para la aplicación de RM

Figura 1: Variables que ejercen una influencia significativa en los obstáculos iniciales y dificultades actuales para aplicar RM

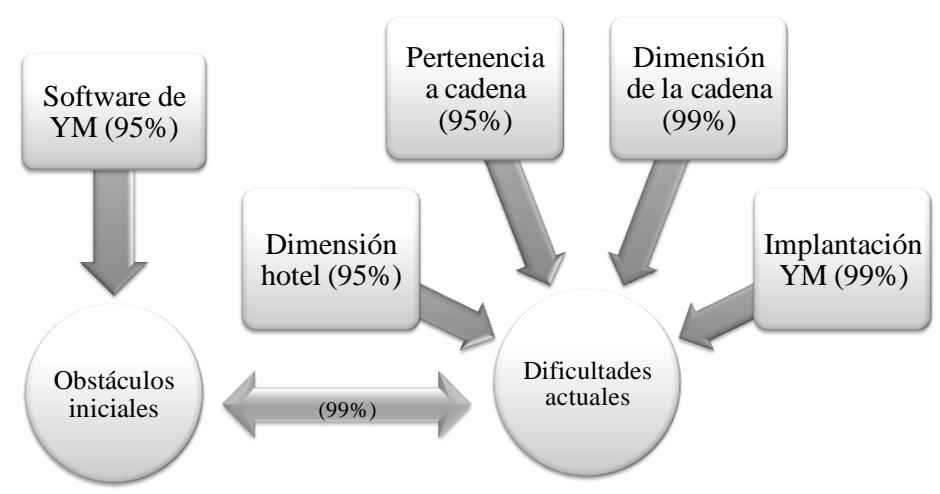


Por otro lado, llama la atención que la influencia de la utilización de software de RM se haya demostrado para las dificultades al inicio del proceso de implantación y no para las barreras o dificultades actuales, lo que confirma la creencia de que, pese a no ser necesario para la implantación de RM, la disponibilidad de software facilita el proceso de adopción de la técnica.

Adicionalmente se ha comprobado que no existe influencia de la disponibilidad de un software sobre las dificultades actuales aunque en este último caso se debe tener en cuenta que el número de respuestas se reduce notablemente (dado que se divide el grupo de hoteles que afirman tener implantado RM entre los que disponen de software y los que no).

Derivado del estudio realizado también podemos decir que también se ha demostrado que no existe influencia de la categoría del hotel, la dimensión del hotel, pertenencia a una cadena, el ámbito en que opera la cadena, la dimensión de la cadena, individualmente considerados, sobre las dificultades que tiene el hotel al iniciar la aplicación de RM. Tampoco influyen la categoría del hotel, el ámbito de operación de la cadena, el conocimiento de los términos YM y RM, sobre las dificultades actuales.

\section{AGRADECIMIENTOS}

Agradecemos a D. Paco Piedras (Director de Formación de Directivos de la Cadena Sol Meliá) sus valiosas aportaciones en el proceso de revisión del cuestionario. Al Dr. Carlos Arias Martín por su orientación en la selección de la muestra objeto de estudio y al Dr. Manuel Rey Moreno por su apoyo e ilusión mostrada en relación con la investigación desarrollada. De forma muy especial, agradecemos a todos los hoteles de cuatro y cinco estrellas de Sevilla y provincia, no solo por su participación en este proyecto sino también por la disposición mostrada a la hora de participar en esta u otras acciones desarrolladas en materia de RM. A todos ellos, dedicamos además los resultados obtenidos en esta investigación.

\section{BIBLIOGRAFÍA}

Brotherton, B.; Mooney, S. (1992). "Yield management: progress and prospects". International Journal of Hospitality Management, 11 (1): 23-32.

Cook, T. (1998). "Sabre soars". OR/MS Today, 25(3): 26-31.

Cross, R.G., Higbie, J.A. y Cross, D.Q. (2009). "Revenue management's renaissance: a rebirth of the art and science of profitable revenue generation". Cornell Hospitality Quarterly, 50(1): 56-81.

Chávez-Miranda, M.E. y Ruiz-Jiménez, A. (2005a): Yield management en hotelería. Estudio de su aplicación en Sevilla. Sevilla: Consorcio de Turismo de Sevilla. Ayuntamiento de Sevilla.

Chávez-Miranda, M.E. (2005b): Yield management. Estudio de su aplicación en el sector hotelero. Universidad de Sevilla. Tesis Doctoral.

(http://fondosdigitales.us.es/tesis/tesis/1940/yield-management-estudio-de-su-aplicacion-en-el-sector-hotelero/).

Comisión Europea (1997): Yield management in small and medium sized enterprises in the tourism industry. European Commission - General Report. European Commission. Directorate-General XXIII, Tourism Unit, Brussels, Luxembourg.

Donaghy, K.; McMahon-Beattie, U. y McDowell, D. (1997). "Implementing yield management: Lessons from the hotel sector". International Journal of Contemporary Hospitality Management, 9 (2): 50-54.

Elimam, A.A. y Dodin, B.M. (2001). "Incentives and yield management in improving productivity of manufacturing facilities". IIE Transactions, 33(6): 449-62.

Flynn, B.B.; Sakakibara, S.; Schroeder, R.G.; Bates, K.A.y Flynn, E.J. (1990). "Empirical research methods in operations management". Journal of Operations Management, 9(2): 250-284.

Geraghty, M. K. y Johnson, E. (1997). "Revenue management saves national car rental”. Interfaces, 27(1): 107127.

González-Fernández, A.M. y Sulé-Alonso, M.A. (1994). "Expectativas de la utilización del yield management en las empresas turísticas". Estudios Turísticos, 123: 47-70.

Guadix-Martín, J. (2004): Gestión de empresas de servicios con yield management. Aplicación en el sector hotelero. Universidad de Sevilla. Tesis Doctoral.

(http://fondosdigitales.us.es/tesis/tesis/215/gestion-de-empresas-de-servicios-con-yield-management-aplicacional-sector-hotelero/).

Jarvis, N.; Lindh, A. y Jones, P. (1998). "An investigation of the key criteria affecting the adoption of yield management in UK hotels". Progress in Tourism and Hospitality Research. 4(3): 207-216. 
Jauncy, S.; Mitchell, I. y Slamet, P. (1995). "The meaning and management of yield in hotels". International Journal of Contemporary Hospitality Management, 7(4): 23-26.

Jones, P. (1999). "Yield management in UK hotels: A system analysis". Journal of Operational Research Society, 50(11) 1111-1119.

Jones, P. y Hamilton, D. (1992). "Yield management: Putting people in the big picture". Cornell Hotel and Restaurant Administration Quaterly, 33(1): 89-95.

Kasilingam, R. (1996). "Air cargo revenue management: Characteristics and complexities". European Journal of Operations Research, 96(1): 36-44.

Kimes, S.E. (1994). "Perceived fairness of yield management". Cornell Hotel and Restaurant Administration Quaterly, 35(1) 22-29.

Kimes, S.E. (1999). "Implementing restaurant revenue management". Cornell Hotel and Restaurant Administration Quarterly, 40(3): 16-21.

Kimes, S.E. (2000): "A strategic approach to yield management". En: Ingold, A.;Yeoman, I. y McMahon-Beattie, U. (Eds) Yield Management: Strategies for the Service Industries (2nd ed.). London: Thomson, pp. 3-14.

Kimes, S.E. (2002). "Perceived fairness of yield management". Cornell Hotel and Restaurant Administration Quaterly. 43(1): 21-30.

Kimes, S.E., Barrash, D.I. y Alexander, J.E. (1999). "Developing a restaurant revenue-management strategy". Cornell Hotel and Restaurant Administration Quarterly, 40(5): 18-29.

Kimes, S.E., Chase, R.B., Choi, S., Lee, P.Y. y Ngonzi, E.N. (1998). "Restaurant revenue management. Applying yield management to the restaurant industry". Cornell Hotel and Restaurant Administration Quarterly, 39(3): 32-39.

Kimes, S.E. y Thompson, G.M. (2004). "Restaurant revenue management at Chevys: determining the best table mix". Decision Sciences, 35(3): 371-92.

Lieberman, W.H. (1993). "Debunking the myths of yield management". Cornell Hotel and Restaurant Administration Quaterly, 34(1): 34-41.

Lippman, B.W. (2003). "Retail revenue management - competitive strategy for grocery retailers". Journal of Revenue and Pricing Management, 2(3): 229-33.

Luciani, S. (1999). "Implementing yield management in small and medium sized hotels: An investigation of obstacles and success factors in Florence hotels". International Journal of Hospitality Management, 18(2): 129-142.

Norman, E.D. y Mayer, K.J. (1997). "Yield management in Las Vegas casino hotels". Cornell Hotel and Restaurant Administration Quaterly, 38(5): 28-33.

Okumus, F. (2004). "Implementation of yield management practices in service organisations: Empirical findings from a mahor hotel group". Service Industries Journal, 24(6): 65-89.

Ortega Martínez, E. y Rodríguez Herráez , B. (2003). "Análisis de contenido sobre la investigación en España y Francia a través de tesis doctorales". Aedem XVII Congreso Nacional, XIII Congreso Hispano-Francés. Burdeos, Francia.

Pinder, J. (2005). "Using revenue management to improve pricing and capacity management in programme management". Journal of the Operational Research Society, 56(1): 75-87.

Relihan, W.J. (1989). "The yield management approach to hotel-room pricing”. Cornell Hotel and Restaurant Administration Quaterly. 30(3): 40-45.

Ryan, C. y Garland, R. (1999). "The use of a specific non-response option on Likert-type scales". Tourism Management, 20(1): 107-113.

Siguaw, J.A., Kimes, S.E.y Gassenheimer, J.B. (2003). "B2B sales force productivity: applications of revenue management strategies to sales management". Industrial Marketing Management, 32(7): 539-551.

Smith, B.C., Leimkuhler, J.F. y Darrow, R. M. (1992). “Yield management at American Airlines”. Interfaces, 22(1): 8-31.

Talón-Ballesteros, P. (2009): "El yield management como filosofía de gestión empresarial. Situación actual en el sector hotelero de la Comunidad de Madrid". Revista del Instituto de Estudios Económicos, 1: 207-28.

Upchurch, R.S.; Ellis, T. y Seo, J. (2002). "Revenue management underpinnings: An exploratory review". International Journal of Hospitality Management, 21(1): 67-83.

Upchurch, R.S.; Ellis, T. y Seo, J. (2003). "A case study of the yield management conundrum: Usage versus competence". Journal of Hospitality and Tourism Research, 27(1): 125-137. 